\title{
On size, radius and minimum degree
}

\author{
Simon Mukwembil| \\ University of KwaZulu-Natal, South Africa. \\ received $9^{\text {th }}$ Feb. 2012, accepted $4^{\text {th }}$ Jan. 2014.
}

Let $G$ be a finite connected graph. We give an asymptotically tight upper bound on the size of $G$ in terms of order, radius and minimum degree. Our result is a strengthening of an old classical theorem of Vizing (1967) if minimum degree is prescribed.

Keywords: interconnection networks, graph, size, radius, minimum degree.

\section{Introduction}

Let $G=(V, E)$ be a finite simple graph. We denote the order of $G$ by $n$ and the size by $m$. The degree of a vertex $v$ in $G$ is denoted by $\operatorname{deg}_{G}(v)$, and the minimum degree of $G$ is denoted by $\delta$. For two vertices $u, v$ in $G, d_{G}(u, v)$ denotes the usual distance between $u$ and $v$ in $G$, i.e., the minimum number of edges on a path from $u$ to $v$. The eccentricity $\operatorname{ec}_{G}(v)$ of a vertex $v$ is the distance from $v$ to a vertex farthest from $v$. The radius $\operatorname{rad}(G)$ of $G$ is the minimum of the eccentricities of the vertices of $G$.

Apart from being an attractive graph parameter, the radius has many practical applications, for instance in network design. If a graph represents a transportation or communication network, then the radius is a measure of the distance a commodity or message has to travel in the worst case, if it is distributed by a single, optimally located source [1]. We are particularly interested in the maximum number of links of a network in which the maximum distance from the optimal source, i.e., the radius of the underlying graph, is limited.

Several upper and lower bounds on the size of a graph in terms of other graph parameters have been investigated. For instance, an upper bound on the size in terms of order and diameter was determined by Ore [3] as early as 1968, while Vizing [4] gave an upper bound in terms of order and radius. Recently, Dankelmann and Volkmann [1] reported lower bounds in terms of order, radius and minimum degree. In this work, we present an upper bound on the size in terms of order, radius and minimum degree. Our bound is a strengthening of Vizing's Theorem [4] which we state below.

${ }^{\dagger}$ Email: mukwembi@ukzn.ac.za. This material is based upon work supported financially by the National Research Foundation.

1365-8050 @ 2014 Discrete Mathematics and Theoretical Computer Science (DMTCS), Nancy, France 
Theorem 1 (Vizing [4]) Let $G$ be a connected graph of order $n$, radius $r$ and size $m$. Then

$$
m \leq \begin{cases}\frac{n(n-1)}{2} & \text { if } r=1, \\ \left\lfloor\frac{n(n-2)}{2}\right\rfloor & \text { if } r=2, \\ \frac{1}{2}(n-2 r)^{2}+\frac{1}{2}(5 n-6 r) & \text { if } r \geq 3 .\end{cases}
$$

We will make use of the well-known handshaking lemma.

Lemma 1 Let $G$ be a graph of size $m$. Then $2 m=\sum_{x \in V(G)} \operatorname{deg}_{G}(x)$.

The notation we use is as follows. $G=(V, E)$ is a connected graph with radius $r, r \geq 9$. For a vertex $v, N_{i}(v):=\left\{x \in V \mid d_{G}(x, v)=i\right\}, i=0,1, \ldots, \mathrm{ec}_{G}(v)$. Let $z$ be a fixed central vertex of $G$, so that $r=\operatorname{ec}_{G}(z)$. For each $i=0,1, \ldots, r$, let $N_{i}=N_{i}(z)$. Hence, $V=N_{0} \cup N_{1} \cup \cdots \cup N_{r}$ is a partition of $V$. We denote by $N_{\leq j}$ and $N_{\geq j}$ the sets $\cup_{0 \leq i \leq j} N_{i}$ and $\cup_{j \leq i \leq r} N_{i}$, respectively. Let $T$ be a spanning tree of $G$ that is distance-preserving from $z$; that is, $d_{T}(v, z)=d_{G}(v, z)$ for all vertices $v \in V$. For vertices $u, v \in V$, let $T(u, v)$ denote the (unique) $u$-v path in $T$. Let $z_{r} \in N_{r}$. We say that a vertex $y \in V$ is related to the vertex $z_{r}$ if there exist vertices $u, v \in V$, where $u \in V\left(T\left(z, z_{r}\right)\right) \cap N_{\geq 9}$ and $v \in V(T(z, y)) \cap N_{\geq 9}$ such that $d_{G}(u, v) \leq 4$.

The following observation is due to Erdös, Pach, Pollack and Tuza [2].

Fact 1 Let $G$ be a connected graph with radius $r \geq 9$ and let $z$ be a central vertex of $G$. For each vertex $z_{r} \in N_{r}(z)$, there exists a vertex in $N_{\geq r-9}$ which is not related to $z_{r}$.

\section{An upper bound on the size}

In this work, we prove the following result which is a strengthening of Vizing's Theorem if minimum degree is prescribed.

Theorem 2 Let $G$ be a connected graph of order $n$, radius $r \geq 9$, minimum degree $\delta \geq 2$ and size $m$. Then

$$
m \leq \frac{1}{2}\left(n-\frac{2 r}{3}(\delta+1)\right)^{2}+(\delta+1)\left(13 n-\frac{22 r}{3}(\delta+1)-r\right)+O\left(\delta^{2}\right) .
$$

Moreover, this bound is asymptotically tight.

Proof: Let $z$ be a fixed centre vertex, $z_{r} \in N_{r}(z)$ and assume the notation of $T$ and $T(u, v)$ above. Let $z_{9}$ be the unique vertex in $V\left(T\left(z, z_{r}\right)\right) \cap N_{9}$ and write $L=T\left(z_{9}, z_{r}\right)=z_{9}, z_{10}, \ldots, z_{r}$. By Fact 1 , let $x \in N_{\geq r-9}$ be a vertex not related to $z_{r}$. Let $T(z, x)=z_{0}, x_{1}, x_{2}, \ldots, x$ and $P=x_{9}, x_{10}, \ldots, x_{r-9}$ be the sub-path of $T(z, x)$ from $x_{9}$ to $x_{r-9}$. Note that since $x$ is not related to $z_{r}$, we have

$$
d_{G}(u, v) \geq 5 \text { for all } u \in V(L) \text { and } v \in V(P) .
$$

Let $L_{1} \subseteq V(L)$ be the set

$$
L_{1}:=\left\{z_{3 i+1} \mid i=3,4, \ldots,\left\lfloor\frac{r-1}{3}\right\rfloor\right\}
$$


For each vertex $v \in L_{1}$, choose any $\delta$ neighbours $u_{1}, u_{2}, \ldots, u_{\delta}$ of $v$ and denote the set $\left\{v, u_{1}, u_{2}, \ldots, u_{\delta}\right\}$ by $M[v]$ and set $L^{\prime}=\cup_{v \in L_{1}} M[v]$.

Similarly, let $P_{1} \subseteq V(P)$ be the set

$$
P_{1}:=\left\{x_{3 i+1} \mid i=3,4, \ldots,\left\lfloor\frac{r-10}{3}\right\rfloor\right\} .
$$

For each vertex $v \in P_{1}$, choose any $\delta$ neighbours $u_{1}, u_{2}, \ldots, u_{\delta}$ of $v$ and, as above, denote the set $\left\{v, u_{1}, u_{2}, \ldots, u_{\delta}\right\}$ by $M[v]$. Set $P^{\prime}=\cup_{v \in P_{1}} M[v]$. Finally, let $M=L^{\prime} \cup P^{\prime}$. Then

$$
|M|=(\delta+1)\left(\left\lfloor\frac{r-1}{3}\right\rfloor+\left\lfloor\frac{r-10}{3}\right\rfloor-4\right) .
$$

Claim $1 \sum_{v \in M} \operatorname{deg}_{G}(v) \leq 2(\delta+1)(2 n-r)+O(\delta)$.

Proof of Claim 1. Note that $\sum_{v \in M} \operatorname{deg}_{G}(v)=\sum_{v \in L^{\prime}} \operatorname{deg}_{G}(v)+\sum_{v \in P^{\prime}} \operatorname{deg}_{G}(v)$. First we consider $\sum_{v \in L^{\prime}} \operatorname{deg}_{G}(v)$. Partition $L_{1}$ as $L_{1}=L_{2} \cup L_{3}$, so that for each $u, v \in L_{i}, i=2,3, d_{G}(u, v) \geq 6$. Precisely, let $L_{2} \subset L_{1}$ be the set $L_{2}=\left\{z_{j} \in L_{1} \mid j \equiv 4(\bmod 6)\right\}$, and $L_{3}=L_{1}-L_{2}$. Write the elements of $L_{2}$ as $L_{2}=\left\{w_{1}, w_{2}, \ldots, w_{\left|L_{2}\right|}\right\}$. For each $w_{j} \in L_{2}$, let $M\left[w_{j}\right]=\left\{w_{j}, u_{1}^{j}, u_{2}^{j}, \ldots, u_{\delta}^{j}\right\}$, where $u_{1}^{j}, u_{2}^{j}, \ldots, u_{\delta}^{j}$ are neighbours of $w_{j}$. The fact that for each $u, v \in L_{2}, d_{G}(u, v) \geq 6$, in conjunction with (1), yields

$$
n \geq\left(\operatorname{deg}_{G}\left(w_{1}\right)+1\right)+\left(\operatorname{deg}_{G}\left(w_{2}\right)+1\right)+\cdots+\left(\operatorname{deg}_{G}\left(w_{\left|L_{2}\right|}\right)+1\right)+\left|P^{\prime}\right|,
$$

and for $t=1,2, \ldots, \delta$,

$$
n \geq\left(\operatorname{deg}_{G}\left(u_{t}^{1}\right)+1\right)+\left(\operatorname{deg}_{G}\left(u_{t}^{2}\right)+1\right)+\cdots+\left(\operatorname{deg}_{G}\left(u_{t}^{\left|L_{2}\right|}\right)+1\right) .
$$

Summing, we get

$$
(\delta+1) n \geq \sum_{v \in\left(\cup_{y \in L_{2}} M[y]\right)} \operatorname{deg}_{G}(v)+(\delta+1)\left|L_{2}\right|+\left|P^{\prime}\right| .
$$

Similarly,

$$
(\delta+1) n \geq \sum_{v \in\left(\cup_{y \in L_{3}} M[y]\right)} \operatorname{deg}_{G}(v)+(\delta+1)\left|L_{3}\right|+\left|P^{\prime}\right| .
$$

Adding (3) and (4), we get

$$
2(\delta+1) n \geq \sum_{v \in L^{\prime}} \operatorname{deg}_{G}(v)+(\delta+1)\left|L_{1}\right|+2\left|P^{\prime}\right| .
$$

Analogously,

$$
2(\delta+1) n \geq \sum_{v \in P^{\prime}} \operatorname{deg}_{G}(v)+(\delta+1)\left|P_{1}\right|+2\left|L^{\prime}\right| .
$$

It follows that

$$
4(\delta+1) n \geq \sum_{v \in L^{\prime}} \operatorname{deg}_{G}(v)+\sum_{v \in P^{\prime}} \operatorname{deg}_{G}(v)+(\delta+1)\left(\left|L_{1}\right|+\left|P_{1}\right|\right)+2\left(\left|P^{\prime}\right|+\left|L^{\prime}\right|\right) .
$$


Therefore,

$$
\begin{aligned}
\sum_{v \in M} \operatorname{deg}_{G}(v) & =\sum_{v \in L^{\prime}} \operatorname{deg}_{G}(v)+\sum_{v \in P^{\prime}} \operatorname{deg}_{G}(v) \\
& \leq 4(\delta+1) n-(\delta+1)\left(\left|L_{1}\right|+\left|P_{1}\right|\right)-2\left(\left|P^{\prime}\right|+\left|L^{\prime}\right|\right) \\
& =4(\delta+1) n-3(\delta+1)\left(\left\lfloor\frac{r-1}{3}\right\rfloor+\left\lfloor\frac{r-10}{3}\right\rfloor-4\right) \\
& =(\delta+1)(4 n-2 r)+O(\delta),
\end{aligned}
$$

and the claim is proven.

Now let $Q=V(G)-M$.

Claim 2 If $v \in Q$, then $\operatorname{deg}_{G}(v) \leq n-(\delta+1)\left(\left\lfloor\frac{r-1}{3}\right\rfloor+\left\lfloor\frac{r-10}{3}\right\rfloor\right)+6 \delta+4$.

Proof of Claim 2. Assume that $v \in Q$. Since $L$ is a shortest path, $v$ can only be adjacent to at most $2 \delta+1$ vertices in $L^{\prime}$. Similarly, $v$ can only be adjacent to at most $2 \delta+1$ vertices in $P^{\prime}$. Note also that from (1), $v$ cannot be adjacent to both a vertex from $L^{\prime}$ and a vertex from $P^{\prime}$. Hence $v$ is adjacent to at most $2 \delta+1$ vertices from $M$. In conjunction with (2), we get

$$
\operatorname{deg}_{G}(v) \leq n-1-|M|+2 \delta+1=n-(\delta+1)\left(\left\lfloor\frac{r-1}{3}\right\rfloor+\left\lfloor\frac{r-10}{3}\right\rfloor-4\right)+2 \delta,
$$

and so the claim is proven.

From [2],

$$
|Q|=n-(\delta+1)\left(\left\lfloor\frac{r-1}{3}\right\rfloor+\left\lfloor\frac{r-10}{3}\right\rfloor\right)+4 \delta+4 .
$$

It follows from Claim 2 that

$$
\begin{gathered}
\sum_{v \in Q} \operatorname{deg}_{G}(v) \leq \\
\left(n-(\delta+1)\left(\left\lfloor\frac{r-1}{3}\right\rfloor+\left\lfloor\frac{r-10}{3}\right\rfloor\right)+4 \delta+4\right)\left(n-(\delta+1)\left(\left\lfloor\frac{r-1}{3}\right\rfloor+\left\lfloor\frac{r-10}{3}\right\rfloor\right)+6 \delta+4\right) .
\end{gathered}
$$

For easy calculation, we use

$$
\left\lfloor\frac{r-1}{3}\right\rfloor+\left\lfloor\frac{r-10}{3}\right\rfloor>\frac{2 r}{3}-6
$$

Thus,

$$
\begin{aligned}
\sum_{v \in Q} \operatorname{deg}_{G}(v) & <\left(n-(\delta+1)\left(\frac{2 r}{3}-6\right)+4 \delta+4\right)\left(n-(\delta+1)\left(\frac{2 r}{3}-6\right)+6 \delta+4\right) \\
& =\left(n-\frac{2 r}{3}(\delta+1)\right)^{2}+\left(n-\frac{2 r}{3}(\delta+1)\right)(22 \delta+20)+120 \delta^{2}+O(\delta) .
\end{aligned}
$$


This, in conjunction with Claim 1 yields

$$
\begin{aligned}
\sum_{v \in V} \operatorname{deg}_{G}(v) & =\sum_{v \in M} \operatorname{deg}_{G}(v)+\sum_{v \in Q} \operatorname{deg}_{G}(v) \\
& <2(\delta+1)(2 n-r)+\left(n-\frac{2 r}{3}(\delta+1)\right)^{2}+\left(n-\frac{2 r}{3}(\delta+1)\right)(22 \delta+22)+120 \delta^{2}+O(\delta) \\
& =\left(n-\frac{2 r}{3}(\delta+1)\right)^{2}+2(\delta+1)\left(13 n-\frac{22 r}{3}(\delta+1)-r\right)+O\left(\delta^{2}\right) .
\end{aligned}
$$

The bound in the theorem follows by an application of Lemma 1 .

Finally, to see that for a constant $\delta$ the bound is asymptotically tight, let $n, r>1, r \equiv 1(\bmod 3)$, and $\delta \geq 2$ be three integers. Let $G_{n, \delta, r}$ be the graph with vertex set $V\left(G_{n, \delta, r}\right)=V_{0} \cup V_{1} \cup \cdots \cup V_{2 r}$, where

$$
\left|V_{i}\right|= \begin{cases}1 & \text { if } i \equiv 0 \text { or } 2(\bmod 3) \\ \delta & \text { if } i=1, \\ n-\frac{2 r}{3}(\delta+1)+\frac{2}{3} \delta-\frac{7}{3} & \text { if } i=2 r-1 \\ \delta-1 & \text { otherwise }\end{cases}
$$

with $u v, u \in V_{i}$ and $v \in V_{j}$, being an edge of $G_{n, \delta, r}$ if and only if $|i-j| \leq 1$. Then

$$
m\left(G_{n, \delta, r}\right)=\frac{1}{2}\left(n-\frac{2 r}{3}(\delta+1)\right)^{2}+O(n \delta)+O\left(r \delta^{2}\right)+O\left(\delta^{2}\right),
$$

and the theorem is proven.

\section{References}

[1] P. Dankelmann and L. Volkmann. Minimum size of a graph or digraph of given radius. Inform. Process. Lett., 109:971-973, 2009.

[2] P. Erdös, J. Pach, R. Pollack, and Z. Tuza. Radius, diameter, and minimum degree. J. Combin. Theory $B, 47: 73-79,1989$.

[3] O. Ore. Diameters in graphs. J. Combin. Theory, 5:75-81, 1968.

[4] V. Vizing. The number of edges in a graph of given radius. Soviet Math. Dokl., 8:535-536, 1967. 
